\title{
Estudo Dopplerfluxométrico das Artérias Uterinas antes e após Inserção do DIU
}

\author{
Color Doppler Flow Analysis of the Uterine Arteries before and after DIU Insertion
}

\author{
Marco Aurélio Martins de Souza ${ }^{1}$, Selmo Geber ${ }^{2}$
}

\section{RESUMO}

\begin{abstract}
Objetivo: averiguar se ocorrem alterações nos parâmetros da dopplervelocimetria em usuárias do dispositivo intra-uterino (DIU) T cobre 380, por meio do estudo das artérias uterinas antes e depois da inserção do DIU em mulheres lactantes e não lactantes.

Métodos: ensaio clínico prospectivo, analitico, com 100 pacientes, em que avaliamos à dopplervelocimetria: índice de resistência (IR), indice de pulsatilidade (IP) e relação sístole/ diástole (SD). As pacientes selecionadas foram submetidas à avaliação pré-inserção do DIU e a novos exames pós-inserção, realizados com 30 dias (um ciclo) e com 90 dias (três ciclos). Resultados: os valores obtidos nas artérias uterinas no grupo antes da inserção do DIU foram de 0,9 para o IR, de 2,4 para o IP e de 10,0 para a relação SD. Os valores 30 dias após a inserção do DIU foram de 0,9 para o IR, de 2,5 para o IP e de 10,7 para a relação SD. Após três ciclos aferimos um valor de 0,9 para o IR, de 2,5 para o IP e de 10,7 para a relação SD. A comparação dos resultados obtidos antes e depois da inserção do DIU mostrou valor $p$ de 0,51 para o IR, de 0,37 para o IP e de 0,51 para a relação $S D$, demonstrando que após a inserção, não houve modificações significativas na dopplervelocimetria.

Conclusões: o uso do DIU T cobre 380 não tem efeito nos indices de dopplervelocimetria das artérias uterinas tanto em pacientes amamentando ou não.
\end{abstract}

PALAVRAS-CHAVE: Dopplervelocimetria. Dispositivo intra-uterino. Artérias uterinas.

\section{Introdução}

O dispositivo intra-uterino (DIU) é o segundo método mais utilizado como planejamento familiar $^{1}$, sendo que mais de 100 milhões de mulheres em todo mundo optam por esta modalidade de anticoncepção, que se tornou mais popular a partir da década de 60. O maior número de usuárias residem na China (cerca de $70 \%$ do total). O DIU é um dos métodos mais utilizado em

${ }^{1}$ Universidade Estadual de Montes Claros - MG;

${ }^{2}$ Departamento de Ginecologia e Obstetricia da Faculdade de Medicina da UFMG

Correspondência:

Marco Aurélio Martins de Souza

Av Dep Esteves Rodrigues 616, sala 203 - Centro

39400-215 - Montes Claros - MG

Tel (38) 3221-1771 - Cel: 9986-8910 - Fax: 3222-8397

e-mail : marcoams@uai.com.br países como Bolivia, Chile e Peru e o seu uso aumentou nas últimas décadas na América Latina. No Brasil, a prevalência do uso do DIU é de $1 \%$, estando entre as mais baixas da região ${ }^{2}$.

O DIU é método que está indicado em pacientes que desejam um anticoncepcional eficaz, prático, de longa duração e prontamente reversível, bastando, para isso, a sua simples retirada. Além disso, mulheres com doenças cardiacas, epilepsia, enxaqueca, hipertensão ou doenças hepáticas podem ter problemas com os métodos hormonais, sendo, assim, candidatas ao uso do DIU. Se levarmos em conta o seu tempo de utilização, podemos considerá-lo como de relativo baixo custo. Por tudo isso, atualmente, vários programas de saúde pública em diferentes regiões do mundo o disponibilizam para uso em planejamento familiar ${ }^{3}$. 
Os efeitos adversos que podem advir da sua utilização são os principais responsáveis pela necessidade de remoção ${ }^{4}$. Os principais efeitos colaterais estão relacionados a alterações no ciclo menstrual, ocasionando menorragia ${ }^{4,5}$, dismenorréia ${ }^{6}$, assim como seu deslocamento inferior, tornando-o mal posicionado ${ }^{7,8}$.

Alguns autores como Colli et al. ${ }^{6}$ e Wang et al. ${ }^{9}$ demonstraram a relação entre a menorragia e a presença do DIU ao descreverem o papel de enzimas lisossômicas na origem desta manifestação clínica. Algumas destas enzimas estão em grande concentração nos macrófagos que, por serem células de defesa, estão em número aumentado na superfície endometrial, quando em contato com o DIU. Estes macrófagos agem contra o dispositivo intra-uterino (reação do tipo corpo-estranho), o que ocasiona invariavelmente morte celular, levando a liberação em excesso de enzimas por se romper a membrana dos lisossomos. Além disso, ocorre maior disponibilidade do precursor das prostaglandinas, o ácido araquidônico, liberado quando há ruptura das membranas celulares. Estes autores compararam mulheres com menorragias associadas ao sangramento uterino disfuncional e as que usavam o dispositivo intra-uterino e que também se queixavam de menorragia. Usaram, como grupo controle, pacientes eumenorréicas ou em amenorréia associada à lactação. Os dados sugeriram que a atividade total das enzimas lisossômicas da classe das fosfatases, particularmente a fosfatase ácida e a N-acetyl-beta-Dglucosaminidase, estavam notadamente elevadas no endométrio exposto ao DIU e no endométrio de mulheres com hemorragia uterina disfuncional quando comparadas com o endométrio de mulheres com história de periodos menstruais completamente normais ou que estavam em pós-parto imediato e amamentando. Nenhuma diferença significativa foi observada em mulheres amamentando e nas com ciclo menstrual normal. Pareceu-lhes, então, que a capacidade do tecido endometrial de sintetizar algumas enzimas estava aumentada nas usuárias do DIU e, assim, correlacionada com a menorragia.

Pan et al. ${ }^{10}$ estudaram, em usuárias do DIU, alterações morfológicas no endométrio relacionadas a hemostasia e menorragia. Sugeriram que o DIU estaria associado à menorragia porque a sua presença ocasionava contração deficiente das arteriolas espirais na camada esponjosa, bem como propiciava a formação deficiente de trombos plaquetários e de fibrinas nestes vasos. No grupo de usuárias que não sangravam abundantemen- te, foram vistas degenerações moderadas e dilatações das arteríolas espirais e muitos trombos, e esta poderia ser a causa da ausência da menorragia neste grupo.

As taxas de remoção do DIU, tanto por incremento do fluxo menstrual quanto por dismenorréia, situam-se em torno de $12 \%$ no primeiro ano e aproximadamente $3,5 \%$ nos seguintes 4 a 10 anos de uso, incluindo-se todos os tipos de DIU ${ }^{11}$. Estes efeitos adversos podem ser originados em conseqüência de alterações na circulação uterina, secundárias ao uso do DIU. Entretanto, se assim ocorre, carece-se de estudos mais detalhados. A menorragia e o sangramento intermenstrual foram importantes em determinar a descontinuidade nas usuárias do DIU com índice de 4,7 vezes por 100 mulheres/ano. A dor pélvica e a infecção foram razões de descontinuidade, respectivamente, em 4,4 e 4,3 vezes por 100 mulheres/ano.

A possibilidade de o DIU interferir nas variações cíclicas modificando os índices da dopplervelocimetria foi sugerida por Jarvela et al. ${ }^{11}$, que demonstraram que existia nítida queda na medida do índice de pulsatilidade (IP) em pacientes usuárias que apresentavam dismenorréia intensa, embora não se tenham evidenciado modificações significativas no índice de resistência (IR).

Frajndlich et al. ${ }^{12}$ realizaram dopplervelocimetria transvaginal das artérias uterinas, e mediram o IR e o IP. No grupo de mulheres que estavam usando o DIU e que tinham sangramento excessivo, eles foram mais baixos do que nos demais grupos. Os autores sugeriram que, na presença de IP menor que 2,0, poderia haver aumento no risco de sangramento uterino anormal.

$\mathrm{Na}$ pesquisa realizada na literatura verificamos que, até o momento, apenas estes dois estudos avaliaram as alterações vasculares em usuárias do DIU, por dopplervelocimetria.

O DIU é muito utilizado em pacientes que estão amamentando, pois não interfere nesta etapa tão importante para o binômio mãe/filho. Por este grupo possuir certas particularidades fisiológicas induzidas pela prolactina, dentre elas a baixa dos níveis estrogênicos, o estudo dopplervelocimétrico nestas pacientes tornou-se interessante ao compará-las com as que não estavam amamentando.

O objetivo de nosso estudo foi avaliar o efeito do uso do DIU e, se o mesmo ocasiona alterações na microcirculação endometrial a ponto de estas serem captadas pela dopplervelocimetria das artérias uterinas. Para isso, estudamos os índices de dopplervelocimetria em dois grupos de pacientes, amamentando e não amamentando, e em dois períodos: 30 e 90 dias após a inserção. 


\section{Pacientes e Métodos}

O estudo realizado foi um ensaio clínico prospectivo, analítico, autopareado. Após a aprovação na Comissão de Ética em Pesquisa do Serviço de Saúde Pública da Universidade Estadual de Montes Claros, UNIMONTES, deu-se prosseguimento ao trabalho. Iniciou-se a seleção das usuárias em abril de 2001, tendo-se realizado o último exame dopplervelocimétrico em janeiro de 2002. Todas as pacientes utilizaram o mesmo tipo de dispositivo $\mathrm{T}$ de cobre 380, e foram examinadas pelo mesmo profissional. As pacientes foram selecionadas à medida que procuravam o ambulatório de planejamento familiar da UNIMONTES, obedecendo aos critérios de inclusão: candidatas ao uso do DIU, sem contra-indicação ao método; pacientes que haviam freqüentado o serviço de planejamento familiar no ambulatório da UNIMOMTES, com prévios exames ginecológicos e citologia oncótica do colo uterino, sem alterações; pacientes que concordaram em participar do estudo e que assinaram o termo de consentimento para pesquisa em seres humanos.

Foram excluídas do estudo aquelas que não assinaram o termo de consentimento, ou que apresentavam doenças passiveis de cursar com alterações vasculares, tais como, hipertensão arterial sistêmica e diabete melito. Também foram excluídas as que apresentaram alguma contra-indicação específica ao método ou que não retornaram na data especificada para a realização dos exames subseqüentes.

Das 100 pacientes iniciais do estudo, 26 foram excluídas e as 74 mulheres que permaneceram receberam o DIU e retornaram para o segundo exame. O principal motivo de exclusão foi o não retorno na data especificada. Das 74,34 (45,9\%) estavam amamentando quando da inserção do DIU, sendo constituídos grupos específicos: lactantes e não lactantes. Do inicial, 44 voltaram para a realização do terceiro e último exame com 90 dias após a inserção, portanto no terceiro ciclo menstrual.

Salientamos a importância de este estudo apresentar amostra autopareada.

A idade das pacientes do grupo variou de 17 a 37 anos, com média igual a 25 anos e desvio padrão igual a quatro anos e meio, mostrando predominio de mulheres jovens, sendo que $51(68,8 \%)$ tinham no máximo 26 anos, estando o maior grupo com 31 mulheres (42\%), com a idade compreendida entre os 21 e 25 anos.

A paridade variou de zero em quatro delas $(5,4 \%)$ a cinco filhos em uma mulher de 35 anos, sendo que a maioria, 43 pacientes, tinha apenas um filho $(58,1 \%)$. A história obstétrica revelou ser o parto normal mais comum $(68,9 \%)$ do que a cesariana.

As pacientes incluídas foram encaminhadas para a realização do primeiro exame de dopplervelocimetria, um ciclo antes da inserção do DIU, no período do quinto ao oitavo dia do ciclo menstrual, formando o grupo pré-DIU. Dentro deste grupo as pacientes foram subdivididas em lactantes e não lactantes. Os exames foram realizados no mesmo horário, entre oito e dez horas da manhã, a fim de se evitarem os efeitos do ritmo circadiano ${ }^{13}$ na circulação uterina. Todos os exames foram realizados pelo mesmo profissional para se evitar possivel viés de efeito interobservador ${ }^{14}$. O retorno para o terceiro exame seguiu as mesmas diretrizes, apenas após o intervalo de três ciclos ou 90 dias nas que apresentavam amenorréia.

Todas as pacientes no momento da realização da dopplervelocimetria estavam com a bexiga vazia, para evitar o efeito da distensão vesical sobre as artérias uterinas ${ }^{15}$. As que estavam em amenorréia lactacional eram encaminhadas sem restrições para o primeiro exame. Neste grupo, repetia-se o exame em 30 e 90 dias após a inserção e se comparava separadamente do grupo de ciclo normal.

Os exames dopplervelocimétricos eram realizados em um mesmo aparelho da marca Synergy Diasonic, de modo triplex colorido, com a técnica padrão com correção do ângulo a $60^{\circ}$, utilizando sempre a mesma sonda de $3,5 \mathrm{MHz}$ para a via abdominal e 7,0 $\mathrm{MHz}$ para a via endovaginal, identificando as artérias uterinas direita e esquerda. Escolhia-se o melhor traçado entre três insonações no nivel do início das artérias uterinas ou em sua bifurcação principal no ligamento cardinal. As artérias eram identificadas com cortes (varreduras) longitudinais e transversais desde a região do colo uterino, orientando-se pela artéria iliaca interna. Como o traçado do aparelho mostrava em média seis a oito grupos de ondas, eram selecionadas no mínimo três das melhores que se mostravam na tela do aparelho, chegando ao numero final por meio da média dos valores.

A documentação fotográfica dos espectros de ondas formados pelo estudo dopplervelocimétrico foi realizada em aparelho de video printer da marca Sony UP 801. Empregamos como parâmetros do fluxo IR, o IP e a relação sístole/diástole (SD) de ambas as artérias uterinas direita e esquerda. Os valores destas variáveis eram obtidos automaticamente pelo software do aparelho de ultra-som e em seguida tabulados e armazenados em computador para posterior análise. 
Uma vez realizado o primeiro exame, as pacientes eram orientadas a retornar ao serviço de planejamento familiar da UNIMONTES para a inserção do DIU no período menstrual e, nas que estavam amamentando e em amenorréia, em qualquer dia. Após a inserção do DIU, orientávamos as pacientes a retornar para ultra-sonografia logo no próximo ciclo menstrual, portanto, aproximadamente com 30 a 35 dias de uso do DIU. A escolha para a realização do exame dopplervelocimétrico recaia no quinto ao oitavo dia após o início da menstruação, como forma de padronizar os dados do estudo. Um retorno seqüencial com 90 dias ou três ciclos menstruais era solicitado para o terceiro e último exame.

Para a estatística, utilizamos o teste $t$ de Student para comparar entre os grupos, as variáveis de interesse, IR, IP e relação SD, bem como o teste não paramétrico de Mann-Whitney/Wilcoxon Kruskal-Wallis (equivalente ao $\chi^{2}$ ) para a comparação entre os subgrupos que estavam e não estavam amamentando. Como ponto de corte para significância, ou seja, nivel necessário para rejeição da hipótese de que o DIU tem efeito nas artérias uterinas, utilizamos o valor $\mathrm{p}<0,05(5 \%)$.

Analisamos separadamente as variáveis de interesse, compreendidas pelo IR, pelo IP e pela relação SD para cada subgrupo de pacientes (amamentando e não amamentando), antes e após a inserção do DIU. Como as variáveis não apresentavam diferenças entre o lado esquerdo e o direito na mesma paciente, seja ela lactante ou não, foram consideradas as medianas das duas artérias (direita e esquerda), para as análises e comparações antes e após a inserção do DIU.

\section{Resultados}

Não observamos diferenças significativas entre os valores da artéria uterina do lado direito e a do lado esquerdo quanto IP, IR e a relação SD. Ressalta-se que este resultado foi obtido nas três avaliações (antes e após a colocação do DIU), tanto no grupo de mulheres que estava amamentando, quanto no grupo sem amamentação.

Comparando-se o grupo de mulheres lactantes com aquelas que não eram, foram verificadas similaridades quanto aos IP, IR e a relação SD. Nesta avaliação obtivemos em lactantes um valor de 0,9 para o IR, e de 0,9 em não lactantes; para o IP, um valor de 2,3 em lactantes e de 2,5 em não lactantes, e para a relação $\mathrm{SD}$, um valor de 8,5 em lactantes e 11,3 em não lactantes.

Os valores para as variáveis de interesse antes da inserção do DIU nas 74 pacientes foram de 0,9 para o IR de 2,4 para o IP e de 10,0 para a relação SD. Os valores para o exame de 30 dias após a inserção foram respectivamente para o IR, IP e relação SD de 0,9, de 2,5 e de 10,7. Os valores no exame de 90 dias nas 44 pacientes que retornaram foram de 0,9 para o IR, de 2,6 para o IP e de 11,6 para a relação SD. A análise estatística revelou não haver diferenças significativas nas séries avaliadas, com um valor $\mathrm{p}$ de 0,51 para o IR, de 0,37 para o IP e de 0,51 para a relação SD (Tabelas 1 e 2).

Tabela 1-Comparação dos índices dopplervelocimétricos das artérias uterinas em pacientes lactantes e não lactantes antes e 30 dias após a inserção do DIU.

\begin{tabular}{|c|c|c|c|c|c|c|}
\hline \multirow[t]{3}{*}{$\begin{array}{l}\text { Índices } \\
\text { dopplervelocimétricos }\end{array}$} & \multirow{3}{*}{$\mathbf{n}$} & \multicolumn{2}{|c|}{$\begin{array}{l}\text { Antes do DIU } \\
\text { Lactação }\end{array}$} & \multicolumn{3}{|c|}{$\begin{array}{l}\text { Depois do DIU } \\
\text { Lactação }\end{array}$} \\
\hline & & Sim & Não & Sim & Não & $p$ \\
\hline & & $n=34$ & $n=40$ & $n=34$ & $n=40$ & \\
\hline idice & 74 & 0,9 & 0,9 & 0,9 & 0,91 & 0,51 \\
\hline dic & 74 & 2,3 & 2,5 & 2,4 & 2,6 & 0,37 \\
\hline Relação sístole/diástole & 74 & 8,5 & 11,3 & 10,8 & 10,7 & 0,5 \\
\hline
\end{tabular}

Teste de Mann-Whitney.

Os valores são medianas.

Tabela 2- Comparação dos índices dopplervelocimétricos das artérias uterinas antes e 90 dias (3 ciclos) após a inserção do DIU.

\begin{tabular}{|c|c|c|c|c|c|}
\hline \multirow{3}{*}{$\begin{array}{l}\text { Índices } \\
\text { dopplervelocimétricos }\end{array}$} & \multicolumn{4}{|c|}{ Avaliação } & \\
\hline & \multicolumn{2}{|c|}{ Pré-DIU } & \multirow{2}{*}{\multicolumn{2}{|c|}{$\begin{array}{l}3 \text { ciclos } \\
\text { pós-DIU }\end{array}$}} & \\
\hline & & & & & \\
\hline & $\mathrm{n}$ & & $\mathrm{n}$ & & $p$ \\
\hline Índice de resistência & 74 & 0,9 & 44 & 0,9 & 0,88 \\
\hline Índice de pulsatilidade & 74 & 2,4 & 44 & 2,5 & 0,38 \\
\hline Relação sístole/diástole & 74 & 10,0 & 44 & 10,7 & 0,68 \\
\hline
\end{tabular}

Após a colocação do DIU nos dois grupos (lactantes e não), observamos os seguintes índices: IR em lactantes pós-DIU de 0,9 e de 0,9 nas não lactantes; IP de 2,4 (lactantes) e 2,6 (não lactantes) e relação SD de 10,8 (lactantes) e de 10,7 (não lactantes).

\section{Discussão}

A presença do DIU na cavidade uterina induz o endométrio a produzir uma série de substâncias vasodilatadoras, que poderiam ocasionar modificações no padrão de fluxo vascular uterino. Zhang et al. ${ }^{16}$ colheram amostras de células endometriais por meio de lavado da cavidade e demonstraram pelo radioimunoensaio que o endométrio de usuárias destes dispositivos mostrava aumento marcante na 
concentração de PGF $2 \alpha$ e tromboxana 2 e estava associado a menorragia e a dismenorréia quando comparado ao endométrio de não usuárias. Huang et al. ${ }^{17}$ compararam as modificações nas artérias espiraladas depois da inserção do DIU por meio de reconstrução tridimensional computadorizada em vários cortes seccionais de espécimes endometriais colhidos sempre na mesma fase do ciclo - neste caso, fase lútea - e observaram que, nas usuárias do dispositivo T de cobre, havia nítida vasoconstrição, com o diâmetro diminuído de 22,7 micrômetros (não usuárias) para 17,9 micrômetros.

Já foi demonstrada por muitos autores a importância da dopplervelocimetria no estudo da circulação uterina em mulheres normais e as modificações do padrão vascular neste órgão associadas a sintomatologias clássicas como a dismenorréia. Dmitrovic ${ }^{18}$ usou o IP para determinar se a dismenorréia estaria associada a modificações no padrão vascular uterino e provavelmente correlacionada a uma excessiva produção de prostaglandinas, as quais levariam a vasoconstrição e isquemia. $\mathrm{O}$ autor concluiu que, na vigência de dismenorréia primária, ocorreu importante aumento nos IP em todo o território vascular estudado, incluindo as artérias uterinas, radiais, arqueadas e espiraladas, quando comparadas com mulheres assintomáticas.

Nossos resultados demonstraram que, antes da inserção do DIU, os valores das variáveis analisadas (IR, IP e relação SD), foram muito semelhantes ao descrito em outros estudos, na mesma fase do ciclo menstrual, isto é, entre o quinto e oitavo dia do ciclo ${ }^{19}$. Estes autores aferiram um valor de 0,9 $\pm 0,1$ para o IR, na primeira fase do ciclo, e Hsieh et al. ${ }^{20}$ mostraram que o valor do IP nesta mesma fase do ciclo foi de 2,8 $\pm 1,2$.

$\mathrm{Na}$ revisão da literatura, apenas dois trabalhos publicados correlacionaram os valores de dopplervelocimetria na resistência das artérias uterinas e o uso do DIU. Jarvela et al. ${ }^{11}$ demonstraram uma nítida queda na medida do IP em pacientes que queixavam de dismenorréia, embora não se tenham evidenciado modificações significativas nos IR. Os exames foram realizados antes da inserção, no sexto e nono dias do ciclo menstrual e no meio da fase lútea, obtendo-se as análises gráficas e os respectivos índices dos vasos uterinos. Entretanto, as pacientes com dismenorréia $(n=5)$, tiveram um IP significativamente menor após a inserção do DIU quando comparadas às que não apresentavam esta queixa antes da inserção, respectivamente de $2,8 \pm 0,5$ versus $2,4 \pm 0,2$. Concluíram que o DIU não modificava o IP, embora a dismenorréia estivesse associada a modificações nestes índices.

Frajndlich et al. ${ }^{12}$ realizaram a dopplervelocimetria por via transvaginal, para avaliar o fluxo das artérias uterinas em usuárias do DIU, utilizando os IR e o IP. Os resultados demonstraram que, no grupo de mulheres com o DIU e que tinham sangramento excessivo, tanto o IR quanto o IP foram significativamente mais baixos do que nos demais grupos. Todas as outras comparações não foram estatisticamente significativas. Sugeriram que na presença de IP menor que 2,0 após o uso do DIU, poderia haver aumento no risco de desenvolver sangramento uterino.

Em um dos estudos citados acima, os autores compararam usuárias sintomáticas, as quais apresentavam menorragia, com um grupo controle representado por não usuárias assintomáticas, não se usando amostra autopareada. A ausência do autopareamento pode não ter eliminado vários fatores de confusão, como idade, paridade, uso de medicações, doenças sistêmicas preexistentes e outros mais.

No presente trabalho, a divisão da amostra em lactantes e não lactantes foi realizada na tentativa de manter os grupos mais homogêneos, portanto, menos sujeitos a fatores de confusão. A comparação entre estes dois subgrupos, tanto antes da inserção do DIU como após, não evidenciou alterações significativas nos indices dopplervelocimétricos (v. Tabela 1).

A primeira comparação foi realizada com um ciclo após a inserção do DIU (30 dias) em todas as 74 pacientes do estudo. Novo exame com três ciclos após a inserção (90 dias) foi realizado e em ambos não se verificaram modificações nos índices de dopplervelocimetria. A repetição do exame com três ciclos foi apropriada para dar consistência aos resultados obtidos no primeiro, visto que tornou-se um tempo suficiente para dar origem às alterações induzidas pelo DIU e assim consubstanciar as conclusões.

Nossos resultados demonstraram que o DIU T de cobre não determina alteração vascular nas artérias uterinas perceptíveis com o uso da dopplervelocimetria, no curto prazo, destituindo-o assim de efeito imediato, como se observa no intervalo de 30 dias após a sua inserção. A lactação com seus prováveis efeitos hormonais não interferiu nos resultados. O exame realizado com 90 dias mostrou que o DIU não interfere nos índices de dopplervelocimetria após este período, reforçando que se existem alterações na microcirculação endometrial pela presença do DIU, estas não são captadas pelo exame em questão. Como o padrão de fluxo avaliado neste trabalho é coincidente com os resultados apresentados na literatura para a mesma fase do ciclo, e como o DIU não os modifica, é improvável antever alterações no fluxo vascular uterino de pacientes, antes do uso do DIU, 
que contra-indiquem o método. Os resultados obtidos não nos permitem inferir sobre a seleção de pacientes para serem ou não usuárias do DIU.

\section{ABSTRACT}

Objective: to observe if there is any alteration in the Dopplervelocimetry of the uterine arteries of nursing and not nursing women after the insertion of the copper T 380 intrauterine device (IUD).

Methods: prospective, analytical, self-paired clinical assay, with 100 patients in whom we evaluated the color doppler flow: resistance index (RI), pulsative index (PI) and systole/ diastole (SD) ratio. Nursing and not nursing women were assessed before IUD insertion as well as 30 days (one cycle) and 90 days (three cycles) after the insertion.

Results: the values obtained in the uterine arteries before insertion were: RI: 0.9, PI: 2.4, and SD ratio: 10.0 . The values 30 days after insertion were: RI: 0.9, PI: 2.5 and SD ratio: 10.7. After three cycles we found a value of 0.9 for RI, 2.5 for $P I$ and 10.7 for SD ratio. The comparison of the results before and after the IUD insertion showed a p value of 0.51 for RI, of 0.37 for PI and of 0.51 for SD ratio, demonstrating that after the insertion of the IUD, there were no significant changes in Dopplervelocimetry. Also, there were not significant differences between nursing and not nursing women, concerning these parameters.

Conclusions: the use of copper T 380 IUD has no effect on the Doppler indices of the uterine arteries of both nursing and not nursing women.

KEYWORDS: Color Doppler flow analysis. Intrauterine device. Uterine arteries.

\section{Referências}

1. Mishell DR Jr. Intrauterine devices: mechanisms of action, safety, and efficacy. Contraception 1998; 58 Suppl:45S-53S.

2. Faúndes A. Recent advances in contraception and family planning. Salud Publica Mex 1992; 34:5-17.

3. Pinter B. Continuation and compliance of contraceptive use. Eur J Contracept Reprod Health Care 2002; $7: 178-83$.

4. Farmer M, Webb A. Intrauterine device insertionrelated complications: can they be predicted? J Fam Plann Reprod Health Care 2003; 29:227-31.

5. Escudero F, Gonzáles GF, Delgadillo L, Villena AE. Factors associated with discontinuation rates of the copper T380A IUD in a Peruvian public hospital. Adv Contracept 1999; 15:303-11.

6. Colli E, Tong D, Penhallegon R, Parazzini F. Reasons for contraceptive discontinuation in women
20-39 years old in New Zealand. Contraception 1999; 59:227-31.

7. Trobough GE. Pelvic pain and the IUD. J Reprod Med 1978; 20:167-74.

8. Cox M, Blacksell SE. Clinical performance of the Nova-T380 IUD in routine use by the UK Family Planning and Reproductive Health Research Network: 12month report. Br J Fam Plann 2000; 26:148-51.

9. Wang IY, Fraser IS, Barsamian SP, et al. Endometrial lysosomal enzyme activity in ovulatory dysfunctional uterine bleeding, IUCD users and postpartum women. Mol Hum Reprod 2000; 6:258-63.

10.Pan J, Yu Y, Chen G. The morphologic changes of endometrial hematostatic reaction in intrauterine devices induced menorrhagia. Zhonghua Fu Chan Ke Za Zhi 1995; 30:526-9.

11.Jarvela I, Tekay A, Jouppila P. The effect of diclofenac on uterine artery blood flow resistance during menstruation in patients with and without a copper intrauterine device. Hum Reprod 1998; 13:2480-3.

12.Frajndlich R, von Eye Corleta H, Frantz N. Color Doppler sonographic study of the uterine artery in patients using intrauterine contraceptive devices. J Ultrasound Med 2000; 19:577-9.

13.Zaidi J, Jurkovic D, Campbell S, Pittrof R, McGregor A, Tan SL. Description of circadian rhythm in uterine artery blood flow during the peri-ovulatory period. Hum Reprod 1995; 10:1642-6.

14.Tekay A, Jouppila P. Intraobserver reproducibility of transvaginal Doppler measurements in uterine and intraovarian arteries in regularly menstruating women. Ultrasound Obstet Gynecol 1996; 7:129-34.

15.Battaglia C, Artini PG, D’Ambrogio G, Galli PA, Genazzani AR. Uterine and ovarian blood flow measurement. Does the full bladder modify the flow resistance? Acta Obstet Gynecol Scand 1994; 73:716-8.

16.Zhang M, Wang HF, Meng $Z$, Huang HY. The effect of copper IUD on microcirculation of blood flow in rabbit uterus. Contraception 1987; 36:677-85.

17.Huang L, Chen L, Chen Q. Three dimensional reconstruction of human endometrial spiral arteries preinsertion and postinsertion of IUD. Zhonghua Fu Chan Ke Za Zhi 1996; 31:523-5.

18.Dmitrovic R. Transvaginal color Doppler study of uterine blood flow in primary dysmenorrhea. Acta Obstet Gynecol Scand 2000; 79:1112-6.

19.Kurjak A, Kupesic-Urek S, Schulman H, Zalud I. Transvaginal color flow Doppler in the assessment of ovarian and uterine blood flow in infertile women. Fertil Steril 1991; 56:870-3.

20.Hsieh YY, Chang FC, Tsai HD. Doppler evaluation of the uterine and spiral arteries from different sampling sites and phases of the menstrual cycle during controlled ovarian hyperstimulation. Ultrasound Obstet Gynecol 2000; 16:192-6.

Recebido em: 9/12/2003 Aceito com modificações em: 19/5/2004 